\title{
DESIGN AND IMPLEMENTATION OF THUNNIFORM ROBOTIC FISH WITH VARIABLE BODY STIFFNESS
}

\author{
Zuo Cui* and Hongzhou Jiang*
}

\begin{abstract}
In nature, undulatory swimmers consume minimum energy by adjusting their body's natural frequency to match the tail-beat frequency. Inspired by this, we study the dynamic model of fish body by considering the body shape and the fluid interactions, and develop a soft thunniform robotic fish. The experimental results show that when the driving frequency is close to the natural frequency, the speed of robotic fish reaches the maximum value, approximately 0.25 body length per second. Moreover, an empty space with a different air pressure is embedded into the body of robotic fish to vary its stiffness, and the natural frequency can be adjusted from 2.0 to $2.8 \mathrm{~Hz}$ by modulating the air pressure. The results of robotic fish with variable stiffness show that the forward speed and acceleration are increased with natural frequency, and a smaller stiffness of caudal fin contributes to a better propulsive performance. We also predict that the peak speed and acceleration can be obtained simultaneously by adjusting the stiffness of both fish body and caudal fin properly. These results reveal that the swimming abilities of robotic fish are closely related to the driving frequency and the stiffness property.
\end{abstract}

\section{Key Words}

Thunniform, robotic fish, viscoelastic body, natural frequency, variable stiffness

\section{Introduction}

Natural fish generate thrust by passing a transverse wave down their body [1], and their striking propulsive performance has inspired a wide range of fish-like robots [2], [3]. For example, Polverino et al. [2] built a robotic fish with many actuated degrees of freedom to mimic the movements of swimming fish. This is a traditional way to design a robotic fish, however, several actuators and sophisticated algorithms are always required to control the body motions. Different from this design method, the soft

* School of Mechatronics Engineering, Harbin Institute of Technology, Harbin 150001, People's Republic of China; e-mail: cuiz@umn.edu, jianghz@hit.edu.cn

Recommended by Prof. Maki Habib

(DOI: 10.2316/Journal.206.2017.2.206-4572) robots have also been developed to duplicate the swimming characteristics of natural fish. Typically, Alvarado [4] designed and fabricated a compliant fish robot driven by an electrical motor. This kind of biomimetic device is simple and robust, which can be modelled as a bending flexible beam [4], [5]. Nevertheless, the capabilities of these soft robotic fish are still far from the swimming performance of real fish. Subsequent studies [6] show that the propulsive abilities are closely related to the mechanical property of fish body and the external fluid environment. Therefore, it is necessary to identify the specific features of swimming fish to promote this study that might benefit the design of fish robot.

Another important aspect, just as clarified by biological experiments, is that the passive stiffness of fish body has significant effects on their swimming performance [6], [7]. Tytell et al. [7] found that given the same actuation, the two-dimensional lamprey can produce different motions by tuning their body stiffness. Besides, Leftwich et al. [8] constructed a flexible tail, and the results from experiments showed that increasing the tail flexibility yields less net force at a certain tail-beat frequency, but the force increases when matching the driving frequency with resonant frequency of the tail. This resonant phenomenon is also found in our previous study of a robotic fish with a planar serial-parallel mechanism [9]. From a biomechanical perspective, swimming fish adjust their natural frequency by stretching elastic components, such as muscles and tendons, to match different tail-beat frequencies.

By integrating biological evidence with engineering practice, the designed robotic fish should be adjusting its body's stiffness to match the natural frequency with the tail-beat frequency during steady swimming. Several robotic fish were ingeniously designed to study the effects of body stiffness on propulsive performance [10], [11]. For example, Park et al. [11] mimicked the endoskeleton structure by connecting rigid segments and compliant segments alternately, and controlled the structure stiffness by compressing the compliant segments. Although this structure provides a way to design a robotic fish with variable stiffness, the size of robotic fish would be changed greatly when the body stiffness changes. Nakabayashi et al. [12] used the variable-effective-length springs to construct the 
flexible fins with variable stiffness, but this application is restricted due to its complexity in adjusting the stiffness. Moreover, the methods to design variable stiffness can also be benefited from other kinds of soft robots or devices. For example, Kawamura et al. [13] developed a kind of laminated sheets that can be compressed by vacuum pump, and the bending resistance of the sheets increases after pumping.

Inspired by these studies, we construct a soft robotic fish adopting the features of thunniform fish, and drive it over a wide range of driving frequencies to explore the resonant vibrations. Based on the Lighthill's elongated body theory, the dynamic analysis of fish body is studied by considering the interactions with fluid flow. The method is similar to that employed in [4], but in our study, we take advantage of the effects of axial force, and provide a novel way to adjust the body stiffness of robotic fish. In detail, a cylindrical chamber is embedded into fish body, and the body stiffness is adjusted by modulating the air pressure inside the chamber. The main contributions of this paper are the realization of robotic fish with adjustable stiffness, and the study of the effects of body/tail stiffness on the propulsive performance. The remainder of the paper is organized as follows. Section 2 establishes a dynamic model of fish body to analyse the natural frequency. Sections 3 and 4 present the fabrication process of thunniform robotic fish and the analysis of experiment results, respectively. The conclusions are presented in Section 5.

\section{Natural Vibrations of Thunniform Fish}

In nature, thunniform fish primarily use their body and caudal fin to produce thrust and move forward [14]. During swimming, the lateral deflection of fish body $h(x, t)$ results in a backward propagating wave with an amplitude envelope, and it is expressed as follows:

$$
h(x, t) \approx\left(c_{0}+c_{1} x+c_{2} x^{2}\right) \sin \left(\omega t-\frac{2 \pi}{\lambda} x\right)
$$

where $\omega$ is the tail-beat frequency, $\lambda$ is the wavelength, $c_{0}$, $c_{1}$ and $c_{2}$ are the coefficients of amplitude envelope. The side and dorsal views of fish body are shown in Fig. 1, and
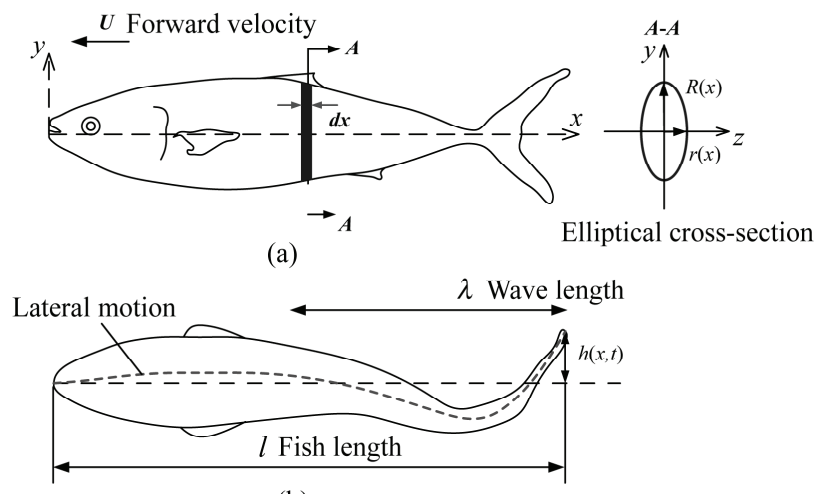

(b)

Figure 1. The side and dorsal views of a thunniform fish. its cross-section can be defined by an ellipse with the major radius $R(x)$ and the minor radius $r(x)$ :

$$
\begin{gathered}
R(x) \approx 0.1 L \sin \left(\frac{2 \pi}{1.57 l} x\right)+0.0008 L \sin \left(e^{2 \pi x / 0.81 l}-1\right) \\
r(x) \approx 0.055 L \sin \left(\frac{2 \pi}{1.25 l} x\right)+0.08 L \sin \left(\frac{2 \pi}{3.14 l} x\right)
\end{gathered}
$$

For swimming fish, the thrust is generated by transferring momentum to the surrounding fluid, and the most relevant model is Lighthill's large-amplitude elongated body theory [15]. In this theory, the thrust force is estimated by the local kinematics at the fish tail, and the lateral force $L_{y}$ exerted on the fish body is analytically expressed as follows:

$$
L_{y}(x) \approx 1.11 m_{a}(x) \frac{\partial^{2} h(x, t)}{\partial t^{2}}
$$

where $m_{a}(x) \approx \pi \rho_{f} R(x)^{2}$ is the added mass of cross-section and $\rho_{f}$ is the fluid density.

In present study, the mechanical properties of fish body are defined by the modulus of elasticity $E(x)$, viscosity $\mu(x)$ and density $\rho(x)$. The cross-sectional area $A(x)$ and the second moment of area $I(x)$ are used to describe the body shape. As shown in Fig. 2(a), when a concentrated harmonic moment $M$ is exerted on the fish body, the flexible body of thunniform fish is deformed as a viscoelastic bending Bernoulli-Euler beam. Here, the natural frequency is analysed to explore the fact that swimming fish actively tune their body stiffness to optimize propulsive performance.

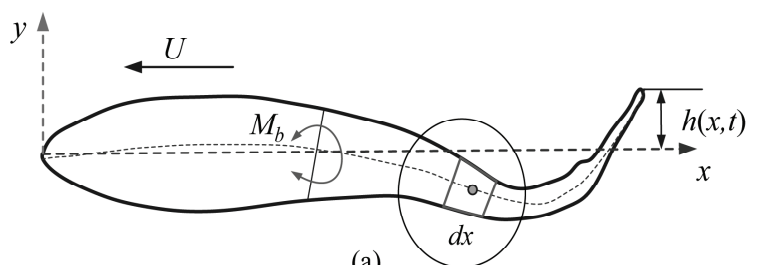

(a)

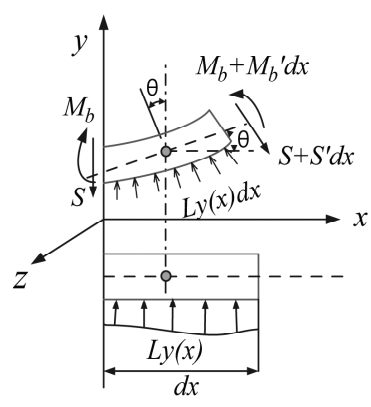

(b)

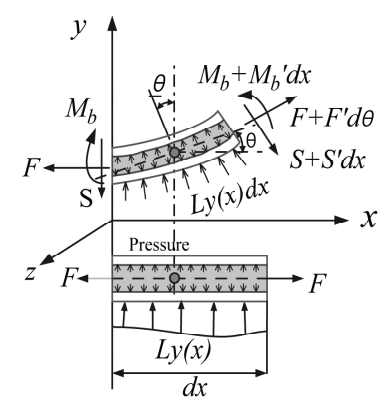

(c)
Figure 2. Dynamic analysis of flexible fish body with/ without axial force: (a) bending fish body actuated by a concentrated moment; (b) bending fish body; and (c) bending fish body with pressure. 


\subsection{Force Analysis of Bending Body without Axial Force}

For small deflection of fish body, the cross-sections remain plane and perpendicular to the centreline after deformation. As shown in Fig. 2(b), the forces acting on body element $d x$ include the shear force $S(x, t)$, the bending moment $M_{b}(x, t)$ and the water-induced lateral force $L_{y}(x, t)$. In Fig. 2(c), $F(\theta)$ is the longitudinal force induced by air pressure. The biological studies [6], [7] suggested that the elasticity, rather than the viscosity, plays a principal role in determining the natural frequency. Therefore, it is acceptable to neglect the effects of viscosity when analysing the natural frequency of fish body. The bending moment $M_{b}$ is a combination of the driving moment $M(x, t)$ and the moment of resistance $M_{e}=E I\left(\partial^{2} h / \partial x\right)^{2}$ due to the elastic body, and expressed as $M_{b} \approx M-M_{e}$. Application of the force balance in the $y$-direction yields:

$$
-\rho A d x \frac{\partial^{2} h}{\partial t^{2}}+S-\left(S+\frac{\partial S}{\partial x} d x\right)-L_{y}(x) d x=0
$$

while the equilibrium of moments yields:

$$
-\left(M_{b}+\frac{\partial M_{b}}{\partial x} d x\right)+M_{b}-L_{y}(x) d x \frac{d x}{2}+S d x=0
$$

The element $L_{y}(x) d x(d x / 2)$ can be neglected due to the small term $d x$, and then combined (4) with (5) and (6), the dynamic equation of fish body is expressed as follows:

$$
\frac{\partial^{2}}{\partial x^{2}}\left(M-E I \frac{\partial^{2} h}{\partial x^{2}}\right)=\rho A \frac{\partial^{2} h}{\partial t^{2}}+1.11 m_{a} \frac{\partial^{2} h}{\partial t^{2}}
$$

Equation (7) models the dynamic behaviours of the fish body moving in a liquid environment in terms of the elastic property, geometry and actuation conditions. However, this equation is difficult to solve by analytical methods, due to the nonlinear changes in both the body shape and the mechanical property [4]. In this study, the fish-like body is simplified as a non-uniform beam with constant elasticity $E$. The cross-sectional area $A(x)$, added mass $m_{a}(x)$ and second moment of inertia $I(x)$ can be roughly expressed as $A_{0} e^{-\lambda x}, m_{a 0} e^{-\lambda x}$ and $I_{0} e^{-\lambda x}$, respectively. However, these approximations are not completely fit with the real shape of fish body, so some refinements are required at the head and tail. For the fish robot, the shape parameters are known, and the materials have homogeneous density and elastic modulus. Therefore, the lateral motion $h(x, t)$ can be assumed as $\Sigma H(x) \sin (\omega t+\varphi)$. When the driving moment $M$ is zero, the natural frequency $\omega$ can be solved by:

$$
H^{\prime \prime \prime \prime}(x)-\frac{\left(\rho A_{0}+1.11 m_{a 0}\right) \omega^{2}}{E I_{0} \lambda^{2}} H(x)=0
$$

For swimming fish, the constraints at the head and caudal fin are free, i.e. both moment and shear force are zero. The natural frequency $\omega_{i}$ of fish body is expressed as follows:

$$
\left[\frac{(i+0.5) \pi}{l}\right]^{4}=\frac{\left(\rho A_{0}+1.11 m_{a 0}\right) \omega_{i}^{2}}{E I_{0} \lambda^{2}}(i=1,2,3 \ldots)
$$

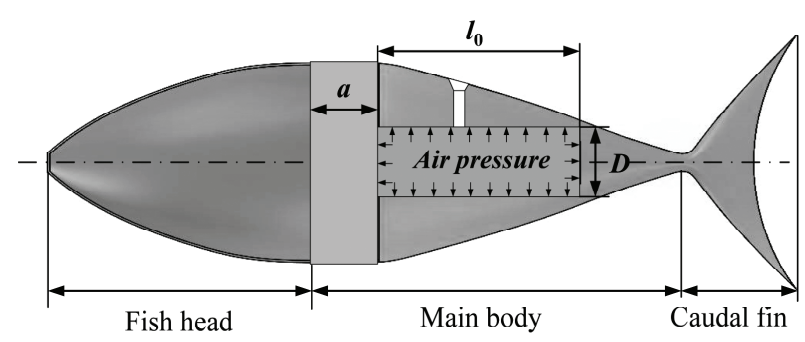

Figure 3. Physical model of the flexible fish body with axial force.

Equation (9) is an approximate solution of the natural frequency of fish body, which is analysed from the dynamic equation of fish body submerged into fluid flow. The results show that the stiffness of fish body $E I_{0}$ is approximately proportional to the square of natural frequency $\omega_{i}^{2}$, and the ratio between the body stiffness and the square of natural frequency is determined by the fish density, the added mass and the cross-sectional area of fish body. In modal analysis, the first mode dominates the motions of swimming fish, and the corresponding natural frequency is written as follows:

$$
\omega_{1}=\sqrt{\frac{E I_{0} \lambda^{2}}{\rho A_{0}+1.11 m_{a 0}}\left[\frac{1.5 \pi}{l}\right]^{4}}
$$

\subsection{Force Analysis of Bending Body with Axial Force}

In our proposed fish model, an empty space is embedded in the body to adjust stiffness by changing air pressure, which is totally different from the traditional soft fish robots. As shown in Fig. 3, the empty space is designed as a cylindrical chamber, which is described by the distance $a$ from fish head to the front of chamber, the cylinder length $l_{0}$ and diameter $D$. The role of air pressure is equivalent to exerting a uniform axial and lateral load, which are distributed to the chamber wall and perpendicular to the surface of the air chamber. This new proposed model can also be regarded as a flexible cantilever beam, but the major difference is the forces induced by air pressure, as shown in Fig. 2(c). The lateral movement of element $(d x)$ satisfies the following equation:

$$
\rho A \mathrm{~d} x \frac{\partial^{2} h}{\partial t^{2}}=-\frac{\partial S}{\partial x} \mathrm{~d} x+\frac{\partial(F \theta)}{\partial x} \mathrm{~d} x-L_{y} \mathrm{~d} x
$$

where $\theta=\partial h / \partial x$ is the rotating angle and $F$ is the axial force induced by air pressure. Substituting (4) into (11), the vibration equation of bending fish body is expressed as follows:

$$
\left(m_{a}+\rho A\right) \frac{\partial^{2} h}{\partial t^{2}}-\frac{\partial}{\partial x}\left(F \frac{\partial h}{\partial x}\right)-\frac{\partial^{2}}{\partial x^{2}}\left(M-E I \frac{\partial^{2} h}{\partial x^{2}}\right)=0
$$

The body motions are also assumed as $H(x) \sin (\omega t+\varphi)$, (12) can be further simplified as follows:

$$
H^{\prime \prime \prime \prime}(x)-\eta^{2} H^{\prime \prime}(x)-\zeta^{2} H(x)=0
$$


where $\eta=\sqrt{F / E I}$ and $\zeta=\sqrt{\left(m_{a}+\rho A\right) \omega^{2} / E I}$. Here, the amplitude envelope $H(x)$ is assumed as $H(x)=$ $c_{1} \cos \left(\lambda_{1} x\right)+c_{2} \sin \left(\lambda_{1} x\right)+c_{3} \cosh \left(\lambda_{2} x\right)+c_{4} \sinh \left(\lambda_{2} x\right)$. The solution coefficient $c_{i}(i=1,2,3,4)$ can be determined by the boundary conditions, and the natural frequency can be derived as follows:

$\omega_{n}=\left(\frac{n \pi}{l_{0}}\right)^{2} \sqrt{\frac{E I}{m_{a}+\rho A}} \sqrt{1+\frac{F}{E I}\left(\frac{n \pi}{l_{0}}\right)^{2}},(n=1,2, \ldots)$

Analysis from (14) reveals that the axial force $F$ produced by the air pressure has a positive relationship with the natural frequency, but the sideways forces $S$ have no effect on it. However, the cross-section of fish body is changed due to the air chamber, which also affects the natural frequency. Overall, (14) provides a theoretical explanation to the mechanism for adjusting body stiffness. It is easy to increase the natural frequency $\omega_{n}$ of the robotic fish by increasing the axial force $F$ or modulating the air pressure inside the chamber.

\section{Soft Robotic Fish without Variable Stiffness}

\subsection{Prototype Design and Fabrication}

For our prototype, the body length from nose to tail is $0.26 \mathrm{~m}$. The body shape is simplified as a streamlined beam with thickness $2 r(x)$ and width $2 R(x)$. The undulation of thunniform fish is mostly confined to the caudal fin with high aspect ratio. During the fabrication process, the distribution of elasticity $E(x)$ of fish body cannot be constructed by casting techniques. Instead, different kinds of soft silicone materials with different moduli can be chosen to make the desired robotic fish. In our experiment, the selected materials comprise a silica gel (hardness Shore $10 \mathrm{~A})$ and an additive that alters the elasticity of the silicone continuously. The elasticity $E$ of fish body is around $37,000 \mathrm{~N} / \mathrm{m}^{2}$, the density of fish body is $1,000 \mathrm{~kg} / \mathrm{m}^{3}$, and the predicted natural frequency is $2.64 \mathrm{~Hz}$.

For the robotic fish, the motor and the crank are embedded into the soft body made of soft silica gel. As shown in Fig. 4, the transmission system of the robotic fish includes an actuator, an actuator support and a rigid plate. The rotational actuator, such as digital servos, is used as the actuation source with different driving frequencies. The flexure linkages connected to the rigid plate are used to transmit the driving torque to the posterior part of fish body. In our robotic fish, the servomotor and electronics are located in the anterior part, and generate a timevarying torque to control the motions of flexible body. In our prototype, the rigid plate is regarded as the ground link, and the arms of servomotor are also acted as two links. During the actuation cycle, the four-bar linkage is used to transmit the external forces, and a cavity is reserved to decrease the mechanical frictions.

In our experiment, the robotic fish is designed to search the natural frequency from a wide range of driving frequencies. As shown in Fig. 5, the actuator (an MG995 steering

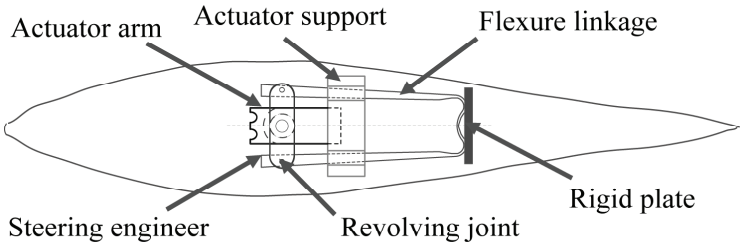

Figure 4. Flexure four-bar transmission and its embedded location in robotic fish.

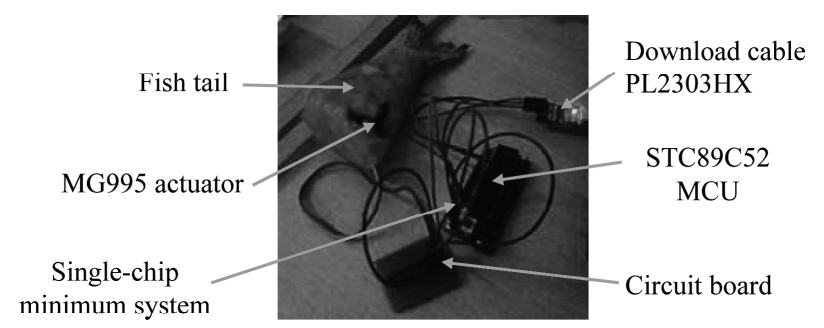

Figure 5. Control hardware with actuation components.

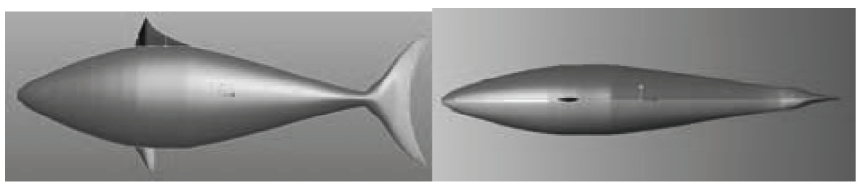

(a)
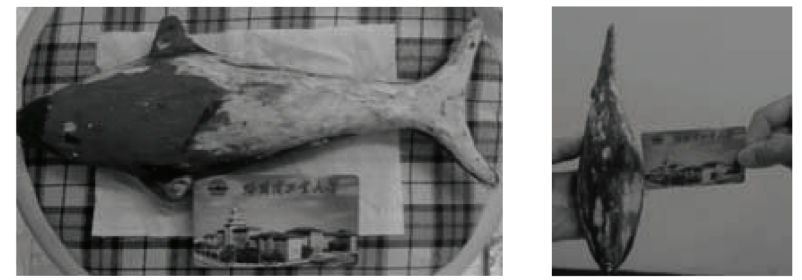

(b)

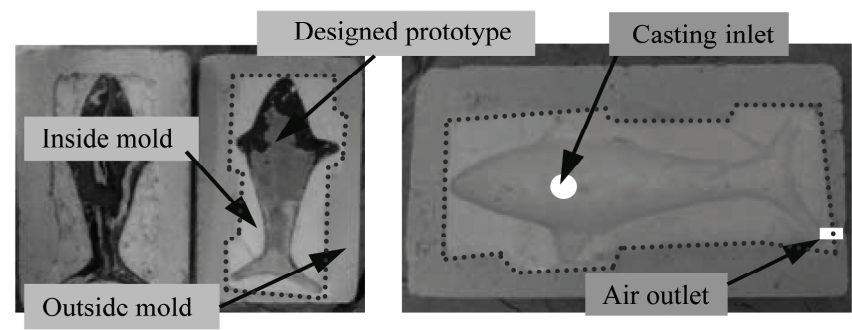

(c)

Figure 6. Procedures of molding the robotic fish: (a) body shape of thunniform fish: side and top; (b) artificial prototype of thunniform fish; and (c) molds for casting the thunniform fish.

gear) can be controlled by the STC89C52 microcontroller unit with an operating program written in $\mathrm{C}++$. The operating program uses a timing circuit in the STC89C52 microcontroller unit to provide a sinusoidal wave. For different frequencies, the operating system is controlled via a wire communication channel, which is connected to the Universal Serial Bus port of a host computer.

Figure 6 shows the molding process to make a robotic fish. At first, an artificial fish with the shape of thunniform 


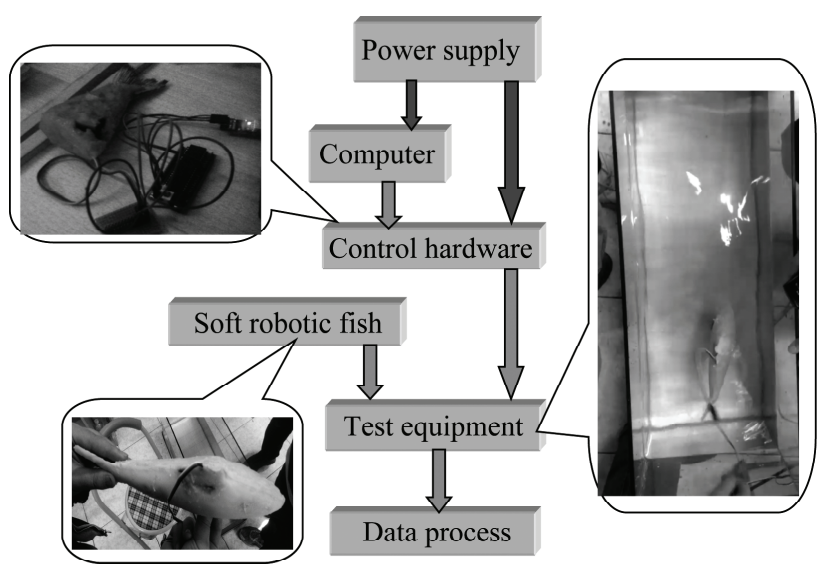

Figure 7. The measurement process of the robotic fish.

fish is carved out from fat lute, and divided into two parts to make the prototype molds. The molds with negative fish shape are made of vulcanized silicone, which can be quickly fabricated in room temperature. The transmission elements, including the actuator, flexure linkage, and rigid plate, are positioned inside a mold before casting. Once the transmission parts are encapsulated inside the molds, two parts of molds are enclosed, and then the silicone rubber is poured in molds to shape the fish body. The mold openings are used for casting the materials and venting the air inside the molds. When the silicone is hardened, the actuation parts inside of body are protected from the water environment. As shown in Fig. 7, the robotic fish with embedded transmission system is controlled via an umbilical cord, which carries the external power and control signals. The experiments are conducted in an uncovered water tank with a size of $0.8 \mathrm{~m} \times 0.5 \mathrm{~m} \times 0.5 \mathrm{~m}$. A high-speed digital camera is adopted to record the instantaneous movement of robotic fish. The collected images are imported to the computer and processed to get more accurate kinematics information.

\subsection{Swimming Performance of Robotic Fish with- out Variable Stiffness}

Operated at different driving frequencies, the robotic fish accelerates from a stationary state until reaching a steady state with constant forward speed. In this experiment, we primarily investigate how the forward speed is affected by the driving frequency. The swimming speed $U$ and the Strouhal number $S t$ are used to evaluate the swimming performance. The Strouhal number is expressed as $S t=f A_{\max } / U$, where $f$ is the driving frequency, and $A_{\max }$ is the lateral amplitude of caudal fin.

In our experiment, the prototype is driven at the same actuation amplitude and different frequencies. As shown in Fig. 8(a), the forward speeds are varied with the driving frequency, which is ranged from 0.5 to $5 \mathrm{~Hz}$ in intervals of $0.5 \mathrm{~Hz}$. When the driving frequency is close to the natural frequency $2.6 \mathrm{~Hz}$, the forward speed reaches the maximum value, 0.25 body lengths $(\mathrm{BL}) / \mathrm{s}$. Our results demonstrate that the swimming speed of robotic fish is largely dependent on the driving frequency and the stiffness

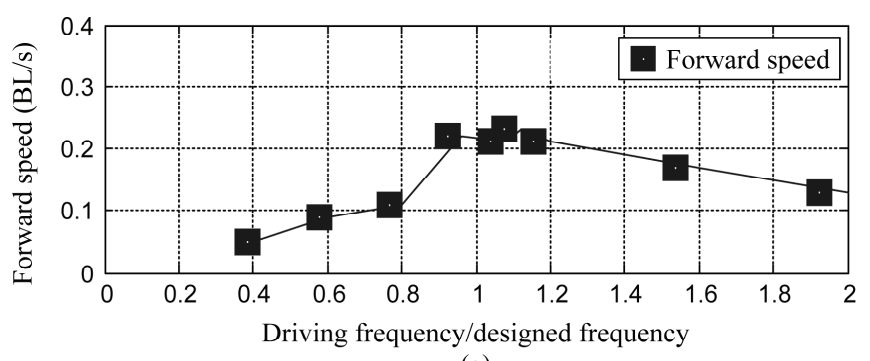

(a)

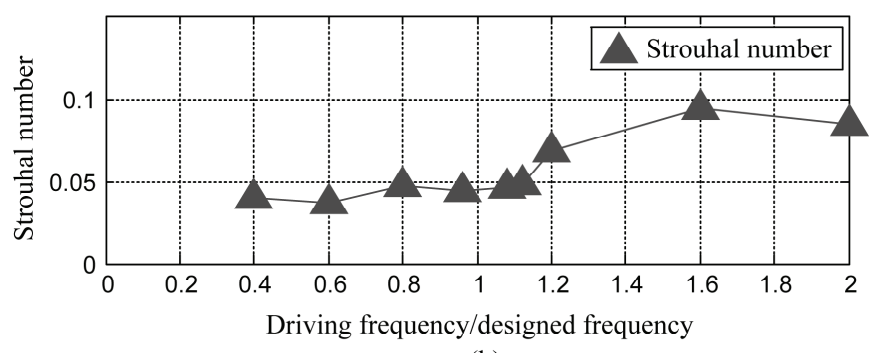

(b)

Figure 8. The trends of (a) speed and (b) Strouhal number varied with driving frequencies.

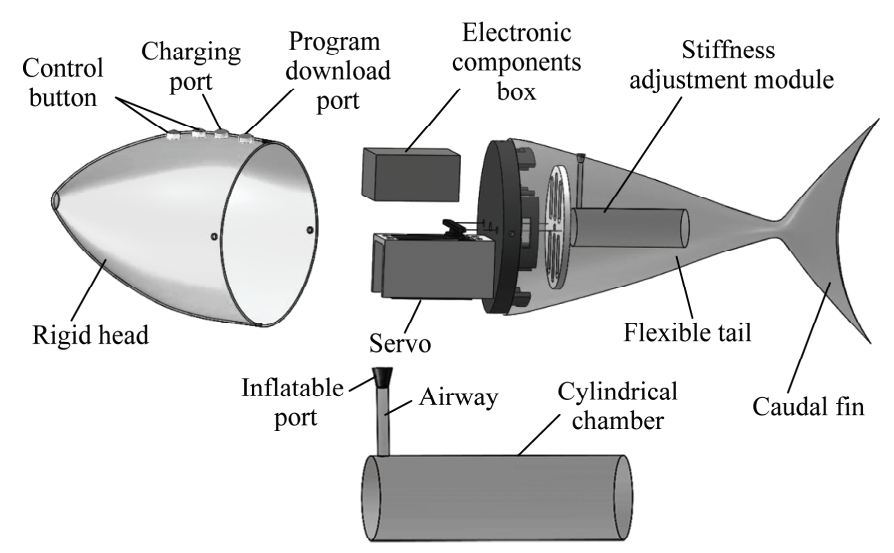

Figure 9. Prototype design of soft robotic fish with variable stiffness.

of fish body. This conclusion is also consistent with the results of experiments done by Alvarado [4]. Meanwhile, this finding makes a contribution in providing a useful method to design the robotic fish optimally. However, as shown in Fig. 8(b), the Strouhal number fluctuates around 0.05 when the driving frequency approaches the natural frequency. This value is much lower than the optimal range $[0.25,0.35]$, meaning that the robotic fish is not as efficient as a real fish.

\section{Soft Robotic Fish with Adjustable Stiffness}

\subsection{Design of Robotic Fish with Adjustable Stiff- ness}

As shown in Fig. 9, we also built the robotic fish with adjustable body stiffness. Compared with the traditional soft fish robots, the different feature of our proposed model is air chamber, in which the air pressure can be changed 
Table 1

The Parameters of Robotic Fish with Adjustable Stiffness

\begin{tabular}{|l|c|c|}
\hline Parts & Parameters & Values \\
\hline \multirow{2}{*}{ Head } & Elasticity & $5 \times 10^{3}(\mathrm{MPa})$ \\
\cline { 2 - 3 } & Length & $150(\mathrm{~mm})$ \\
\hline \multirow{2}{*}{ Body } & Elasticity & $0.061(\mathrm{MPa})$ \\
\cline { 2 - 3 } & Length & $220(\mathrm{~mm})$ \\
\hline \multirow{3}{*}{ Air chamber } & $a$ & $110(\mathrm{~mm})$ \\
\cline { 2 - 3 } & $D$ & $25(\mathrm{~mm})$ \\
\cline { 2 - 3 } & Length & $60(\mathrm{~mm})$ \\
\hline
\end{tabular}

to adjust the body stiffness. The air module consists of a cylindrical chamber, an airway and an inflatable port. In the prototype, the chamber axis is overlapped with the centreline of fish body, and the space between cylindrical front facet and fish head is used to place the actuation and transmission modules. The cylindrical chamber is charged with external air though the inflatable port, and the stiffness of fish body varies with the air pressure. In this prototype, we firstly construct a plastic head shell using 3D print technology, and then make the soft body from liquid silicone $(E=0.061 \mathrm{MPa})$. The shells of rigid head and the soft body are connected by joint parts. Several parameters, as listed in Table 1, should be considered for designing the robotic fish. Besides, we also design a pressure measuring device, which is refitted from a mercury barometer. The pressure value could be measured by synchronizing the aeration, and its measurement range is $0-40 \mathrm{kPa}$.

\subsection{Swimming Performance of Robotic Fish with Adjustable Stiffness}

As known, it is unrealistic to change the natural frequency of the soft robot by constructing several robotic fish with different kinds of materials. However, for our latter model, the bending rigidity of fish body varies with the air pressure of the chamber. Although (14) provides a theoretical explanation to calculate the natural frequency, however, it is difficult to be solved analytically due to the embedded servomotor, linkages, battery and air chamber. Here, we regard that the robotic fish have the maximum forward speed when the driving frequency is close to the natural frequency. Therefore, we charged the robotic fish with a different air pressure (relative to the atmospheric pressure), from 0 to $25 \mathrm{kPa}$ at the interval of $5 \mathrm{kPa}$. Then, the forward speeds were measured when the driving frequency ranged from 1.0 to $3.2 \mathrm{~Hz}$ with the intervals of $0.1 \mathrm{~Hz}$. As shown in Fig. 10, for a different air pressure, the forward speed increases with the driving frequency until reaching a maximum value, then decreases slightly with the driving frequency. The results show that the natural frequency is varied with the air pressure, and ranged from 2.0 to $2.8 \mathrm{~Hz}$. The maximum speed is linearly increased with the natural frequency. We might treat the natural frequency at the air

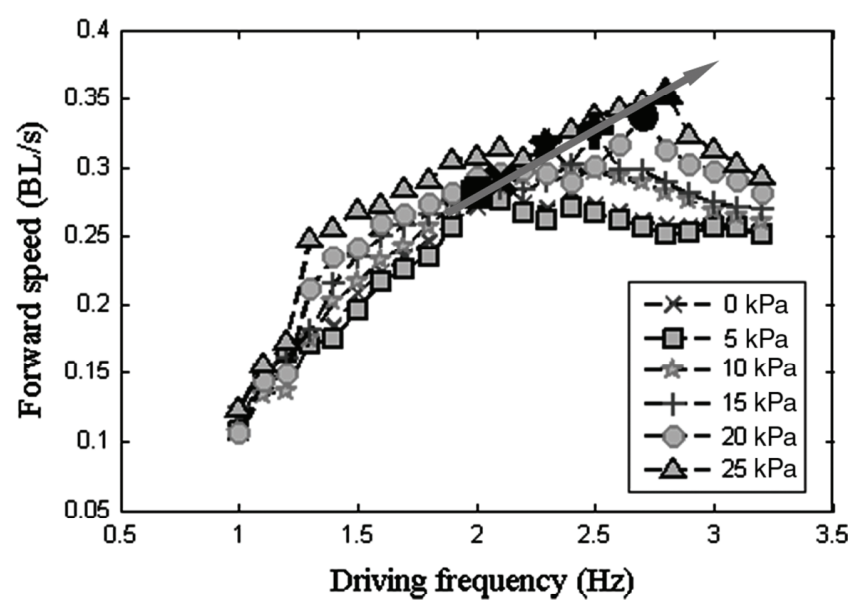

Figure 10. Effect of body stiffness and driving frequency on the swimming speed.

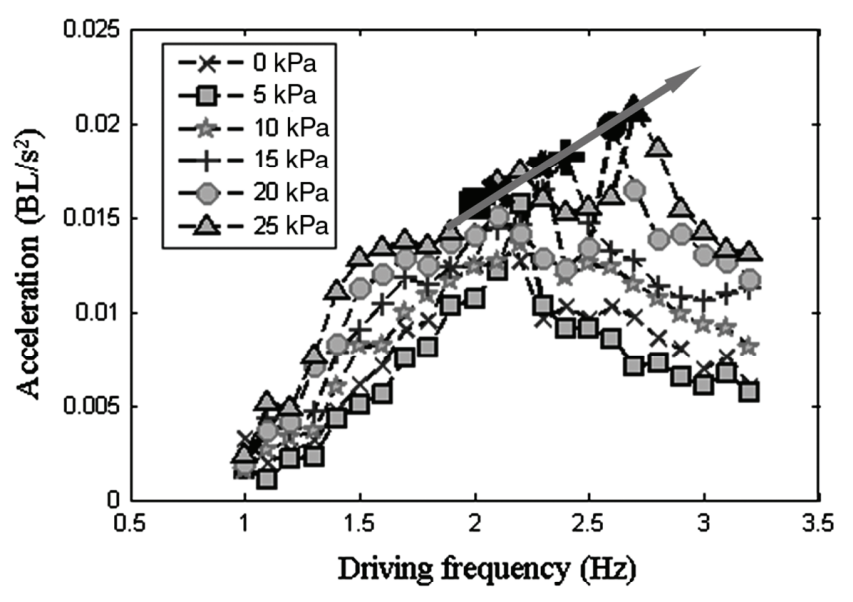

Figure 11. Effect of body stiffness and driving frequency on the swimming acceleration.

pressure value being zero as the initial natural frequency, and use (14) to predict the natural frequency of robotic fish with a different air pressure.

We also measured the acceleration of robotic fish with adjustable stiffness under different driving frequencies, and the results are showed in Fig. 11. Similarly, the maximum acceleration is also increased with the natural frequency. It is notable that the maximum acceleration happens when the driving frequency is close to the natural frequency, but they are not equal, which is different with the speed case. This phenomenon is also pointed out in [10]. For the application, the high speed and acceleration can be obtained when the driving frequency is close to the natural frequency. Overall, changing the driving frequency and/or the body stiffness is a basic approach to improve the swimming performance of robotic fish. In contrast to the limited changing range of actuator's driving frequency, changing the body stiffness is an easy and critical way to satisfy the condition of resonant vibration, and that is the reason why bionics researchers focus on the applications of variable stiffness in swimming fish.

For thunniform fish, the thrust is mainly produced by the caudal fin, and directly affected by the stiffness of 


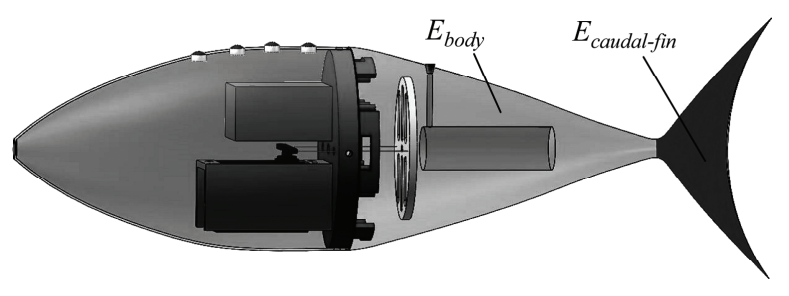

Figure 12. Elasticity distribution of robotic fish with adjustable stiffness.

Table 2

Parameters of Two Prototypes with Adjustable Stiffness

\begin{tabular}{|l|c|c|c|c|}
\hline Prototypes & $\begin{array}{c}E_{b} \\
(\mathrm{MPa})\end{array}$ & $\begin{array}{c}E_{c} \\
(\mathrm{MPa})\end{array}$ & $\begin{array}{c}P \\
(\mathrm{kPa})\end{array}$ & $\begin{array}{c}W_{\mathrm{n}} \\
(\mathrm{Hz})\end{array}$ \\
\hline Fish (a) & 0.061 & 10 & 0 & 2.3 \\
\hline Fish (b) & 0.061 & 0.061 & 0 & 2.1 \\
\hline Fish (b) & 0.061 & 0.061 & 10 & 2.3 \\
\hline
\end{tabular}

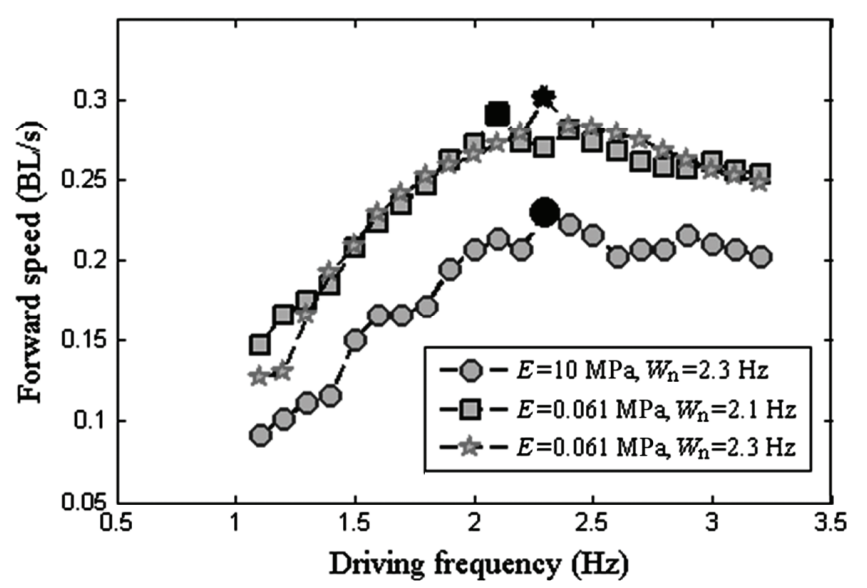

Figure 13. Effect of the caudal fin stiffness on swimming speed.

the caudal fin. To investigate the influences of the caudal fin stiffness on the swimming performance, we build two different prototypes with a different caudal fin, named as Fish (a) and Fish (b), and they are made of liquid silicone with $E=10$ and $0.061 \mathrm{MPa}$, respectively. In the experiment, when air pressure value is 0 , the natural frequency of robotic Fish (a) and robotic Fish (b) is 2.3 and $2.1 \mathrm{~Hz}$, respectively. To study the influences of the stiffness of caudal fin, we change the natural frequency of Fish (b) into $2.3 \mathrm{~Hz}$ after by adjusting the air pressure to $10 \mathrm{kPa}$. For these two prototypes, as shown in Fig. 12, the elastic modulus of fish body $E_{b}$, the elastic modulus of caudal fin $E_{c}$, and their natural frequencies are listed in Table 2.

As shown in Figs. 13 and 14, the forward speed and acceleration of robotic Fish (a) in pressure value 0 and robotic Fish (b) in pressure value 0 and $10 \mathrm{kPa}$ were measured, respectively. In Fig. 14, although the natural

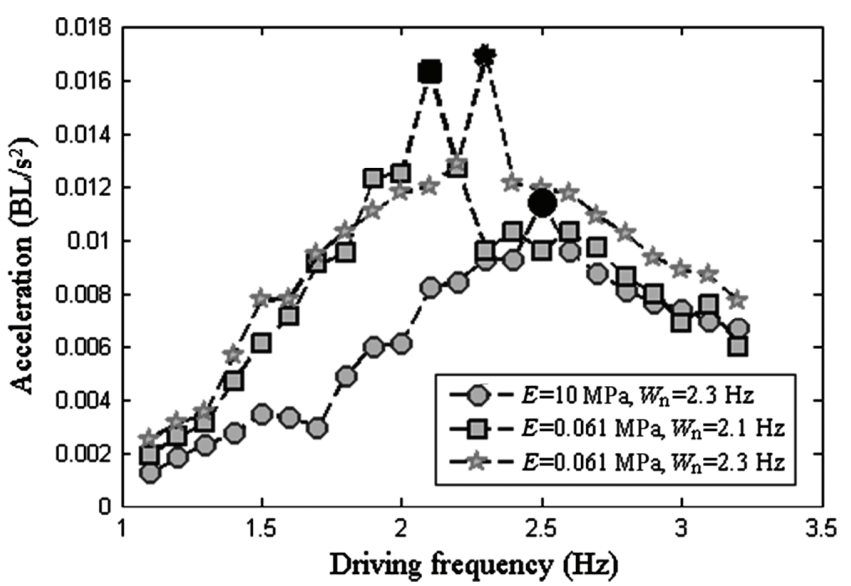

Figure 14. Effect of caudal fin stiffness on swimming acceleration.

frequencies of the robotic Fish (a) and Fish (b) are the same, $2.3 \mathrm{~Hz}$, the driving frequency is different to get the maximum acceleration. The results also show that both the forward speed and acceleration are largely affected by the stiffness of caudal fin, and the smaller stiffness of caudal fin contributes to a larger speed and acceleration. These results indicate that the maximum acceleration of robotic fish is determined by both the body stiffness and the stiffness of caudal fin. Therefore, we predict that in real fish, an optimal stiffness of caudal fin may exist to match with the body stiffness for obtaining the peak forward speed and acceleration simultaneously.

\section{Conclusion}

Different from the traditional fish robots, this paper provides an effective method to design the robotic fish with adjustable stiffness, and two different types of robotic thunniform fish (with or without variable stiffness) were designed and fabricated. In the experiments, we found that when the driving frequency is close to the natural frequency of robotic fish body, the forward speed will reach maximum, about $0.25 \mathrm{BL} / \mathrm{s}$. Further, the natural frequency of robotic fish was adjusted from 2.0 to $2.8 \mathrm{~Hz}$ by charging the air into fish body with a different pressure. The results showed that both swimming speed and acceleration of robotic fish are increased with the natural frequency. Besides, we developed two prototypes with a different caudal fin to study the influences of caudal fin stiffness, and found that lower stiffness of caudal fin contributes to a faster speed and larger acceleration. Therefore, it is reasonable to suppose that the maximum speed and acceleration of robotic fish can be achieved simultaneously by selecting the right stiffness of both fish body and caudal fin. For designing a soft fish robot in the future, CPGs [16] and tracking controls [17] may be adopted for generating the required rhythmic, because the flexible body of our current robot cannot be deformed as the natural fish.

\section{Acknowledgement}

This work was financially supported by the National Natural Science Foundation of China (No. 51275127). 


\section{References}

[1] G.V. Lauder, Fish locomotion: Recent advances and new directions, Annual Review of Marine Science, 7, 2015, 521-545.

[2] G. Polverino, P. Phamduy, and M. Porfiri, Fish and robots swimming together in a water tunnel: Robot color and tailbeat frequency influence fish behavior, PLoS ONE, 8(10), 2013, e77589.

[3] H. Xie, H. Zhou, L. Shen, and D. Yin, Mechanism design, dynamics modelling and experiments of bionic undulating fins, International Journal of Robotics and Automation, 31(2), 2016, $146-155$.

[4] P.V. Alvarado, Design of biomimetic compliant devices for locomotion in liquid environments, Ph.D. Thesis, Massachusetts Institute of Technology, Cambridge, USA, 2007.

[5] P.L. Guyen, V.P. Do, and B.R. Lee, Dynamic modeling of a non-uniform flexible tail for a robotic fish, Journal of Bionic Engineering, 10, 2013, 201-209.

[6] J.H. Long, N.M. Krenitsky, S.F. Roberts, J. Hirokawa, J. de Leeuw, and M.E. Porter, Testing biomimetic structures in bioinspired robots: How vertebrae control the stiffness of the body and the behavior of fish-like swimmers, Integrative and Comparative Biology, 51(1), 2011, 158-175.

[7] E.D. Tytell, C.-Y. Hsu, T.L. Williams, A.H. Cohen, and L.J. Fauci, Interactions between internal forces, body stiffness, and fluid environment in a neuromechanical model of lamprey swimming, Proceedings of the National Academy of Sciences, 107, 2010, 19832-19837.

[8] M.C. Leftwich, E.D. Tytell, A.H. Cohen, and A.J. Smits, Wake structures behind a swimming robotic lamprey with a passively flexible tail, Journal of Experimental Biology, 215, 2012, 416-425.

[9] Z. Cui and H.Z. Jiang, A study of the planar serial-parallel mechanism with various stiffness for a biotic compliant fish, Proc. ASME 2013 International Mechanical Engineering Congress 83 Exposition, San Diego, CA, 2013, 1-6.

[10] A.K. Kancharala and M.K. Philen, Study of flexible fin and compliant joint stiffness on propulsive performance: Theory and experiments, Bioinspiration \& Biomimetics, 9, 2014, 036011.

[11] Y.J. Park, T.M. Huh, D. Park, and K.J. Cho, Design of a variable-stiffness flapping mechanism for maximizing the thrust of a bio-inspired underwater robot, Bioinspiration 83 Biomimetics, 9, 2014, 036002

[12] M. Nakabayashi, R. Kobayashi, S. Kobayashi, and H. Morikawa, Bioinspired propulsion mechanism using a fin with a dynamic variable-effective-length spring: Evaluation of thrust characteristics and flow around a fin in a uniform flow, Journal of Biomechanical Science and Engineering, 4(1), 2009, 82-93

[13] S. Kawamura, T. Yamamoto, D. Ishida, T. Ogata, Y. Nakayama, O. Tabata, and S. Sugiyama, Development of passive elements with variable mechanical impedance for wearable robots, IEEE Int. Conf. Robotics and Automation, Washington, DC, 2002, 248-253.
[14] J.J. Videler, Fish swimming (London, UK: Chapman \& Hall, 1993).

[15] M.J. Lighthill, Large-amplitude elongated-body theory of fish locomotion, Proceedings of the Royal Society of London Biological Sciences, 179(1055), 1971, 125-138.

[16] M.K. Habib, K. Watanabe, and K. Izumi, Biped locomotion using CPG with sensory interaction, IEEE Int. Symp Industrial Electronics, Seoul, 2009, 1452-1457.

[17] F. Sun, J. Yu, P. Zhao, and D. Xu, Tracking control for a biomimetic robotic fish guided by active vision, International Journal of Robotics and Automation, 31(2), 2016, 137-145.

\section{Biographies}

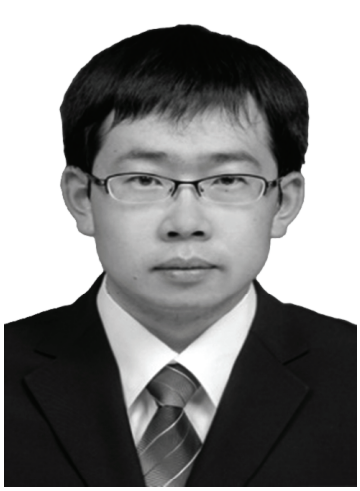

Zuo Cui received his MS degree in Mechanical Engineering from Harbin Institute of Technology, China, in 2013, and currently, he is a joint Ph.D. candidate in Harbin Institute of Technology and University of Minnesota, Twin Cities. His research interest covers robotics and computational fluid dynamics.

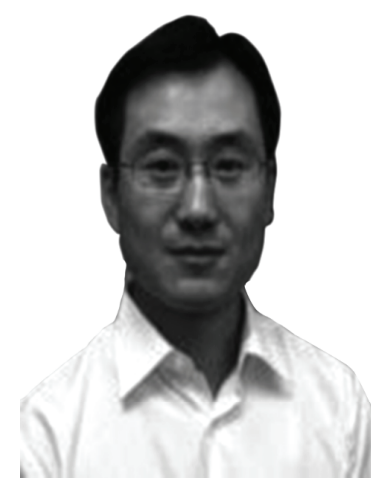

Hongzhou Jiang received his Ph.D. degree in Mechanical Engineering from Harbin Institute of Technology, China, in 2001. Currently, he is a professor in the Department of Mechanical Engineering at Harbin Institute of Technology, China, and a member of Chinese Society of Astronautics. He has published about 70 refereed journal and conference papers. His research interest covers parallel robotics, control systems and hydrodynamic control system. 